\title{
TUMOR MARKERS IN HEMODIALYZED PATIENTS
}

\author{
Alexandra Gireada ${ }^{1}$, Nicolae Bacalbasa ${ }^{2}$, Irina Balescu ${ }^{3}$, Cristian Balalau ${ }^{4}$, Mihai Dimitriu $^{2}$ \\ 1 "Carol Davila" Central Military Emergency Hospital, Bucharest \\ 2 "Carol Davila" University of Medicine and Pharmacy, Bucharest \\ 3 "Ponderas" Hospital, Bucharest \\ 4 "Sf. Pantelimon" Emergency Hospital, Bucharest
}

\begin{abstract}
The issue of serum concentrations of tumor markers in hemodialyzed patients is a widely discussed one due to the fact that these patients are usually at high risk of developing concurrent malignancies on one hand and due to the fact that half-time of these markers is strongly influenced by an impaired renal function. This is a literature review focusing on the role of determination of tumor markers such as CA 125, CA 15-3, AFP in hemodialyzed patients.
\end{abstract}

Keywords: dialysis, CA 125, CA 15-3, AFP

Abbreviations: $\mathrm{HD}=$ hemodialysis

\section{INTRODUCTION}

The use of the tumor markers in dialysis patients is a largely debated subject in the literature because the candidates for transplantation and hemodialysis are persons with a high risk for developing malignancies and because the half-time of the serum tumor markers' concentrations depends upon the renal and hepatic function. (1-3)

There were also noted important fluctuations of some of the tumor markers' concentrations after the HD sessions. Many studies have been done on this theme. One of them, realized by G. Tzitzikos et al in 2010, found that the levels of AFP didn't have significant variations and that the elevations of CA 15-3 and CA 125 levels were associated with active hepatitis $\mathrm{C}$ infection and not with neoplasms. (4)

In order to avoid unnecessary diagnostic procedures in lung cancer patients who also suffer from renal dysfunction, a study realized by F Nomura et al measured the serum concentrations of tumor markers: CYFRA 21-1, ProGRP, CEA, SCC and NSE. The results showed that they were influenced by the degree of renal failure, except from NSE. It is known that ProGRP is a tumor marker with a mo- lecular weight comparable to that of beta2-microglobulin. In this study, the Pro GRP levels decreased after hemodialysis. (5)

Some studies showed that, unlike CEA, CA 19-9 and CA 50 have the same clinical relevance in patients diagnosed with chronic renal failure and patients without this disease. (6)

\section{Determination of tumor markers' concentration in hemodialysed patients with concurrent malignancies}

The measurement of tumor markers' concentrations in uraemic patients is very important because there are some malignancies, like prostate cancer, kidney and corpus uteri cancer, which have a higher incidence in dialysis patients. This fact was demonstrated by Port et al in a well-designed controlled study. (7)

A study realized in 1991 by Cases A. et al included 30 patients diagnosed with chronic renal failure and 36 hemodialyzed patients. These patients had no sign of neoplasia. The authors measured the concentrations of CEA, CA 125, CA 15-3, CA 19-9, CA 50, AFP, NSE, SCC, PSA and pros-

Corresponding author:

Nicolae Bacalbasa, MD, 2 Dimitrie Racoviță Street, Bucharest, Romania

E-mail: nicolae_bacalbasa@yahoo.ro 
tatic acid phosphatase. The percentages of serum increased concentrations in the two groups of patients were $33 \%$ and $47 \%$ for CEA, $37 \%$ and $44 \%$ for CA $50,43 \%$ and $72 \%$ for SCC, $18 \%$ in patients with IRC for CA 125, 36\% in HD patients for NSE. The authors concluded that four tumor markers show false positivities in uremic patients: CEA, SCC, CA 50 and NSE. (8)

\section{Modifications of tumor markers' concentrations in patients submitted to dialysis}

A study realized by W.C. Lye included 30 patients on hemodialysis, 30 patients on peritoneal dialysis, 30 patients with renal transplant and 30 normal controls. The authors measured the concentrations of AFP, CEA, CA 125, CA 19-9 and PSA. The results showed normal concentrations of AFP and PSA in the dialysis and transplant patients. The concentrations of CEA, CA 125 and CA 19-9 were increased in $37 \%, 10 \%$ and $53 \%$ of hemodialysis and increased in $17 \%, 27 \%$ and $57 \%$ of peritoneal dialysis patients. This fact can be explained by their large molecular weight. They were normal in transplant patients and controls, even they were higher in the transplant patients compared to controls. The authors concluded that CEA, CA 125 and CA 19-9 shouldn't be used in dialysed patients diagnosed with malignancies (9).

A study realized in 2000 by A. Sevinc et al included 26 patients on chronic hemodialysis without the presence of serosal fluid and 13 patients on chronic hemodialysis, having serosal fluid in one of the cavities: peritoneum, pleura or pericardium. The study included also 52 healthy volunteers. The only category of patients in which the levels of CA 125 were significantly raised was that of hemodialysis patients without serosal fluids. The levels in the other two groups of patients were similar. The authors concluded that the utilization of CA 125 in hemodialysis patients can be impaired in the presence of serosal fluids. (10)

The use of tumor markers in dialysed patients is necessary because there are some malignancies that are more frequently encountered in these patients than in the general population. However, their values are generally different from those encountered in the general population. A study realized in Japan intended to establish some cut-off values for some tumor markers and for this group of patients. It included 173 HD patients without malignant or benign conditions which could influence the serum levels of the tumor markers and included also 100130 control subjects. The cut-off levels determined were as follows: 131-707 ng/ml for EL-1 (100-400 $\mathrm{ng} / \mathrm{ml}$ in normal individuals), $71 \mathrm{U} / \mathrm{ml}$ for CA-50 (35 U/ml in normal individuals), $76 \mathrm{U} / \mathrm{ml}$ for $\mathrm{CA}$ $19-9$ (37 U/ml in normal individuals), $166 \mathrm{ng} / \mathrm{ml}$ for BFP (75 ng/ml in normal individuals), $45 \mathrm{U} / \mathrm{ml}$ for SLX (30 U/ml in normal individuals), $6.9 \mathrm{ng} / \mathrm{ml}$ for CEA (2.5 ng/ml in normal individuals), 1,068 $\mu \mathrm{g} / \mathrm{ml}$ for IAP $1(500 \mu \mathrm{g} / \mathrm{ml}$ for normal individuals $), 6.5 \mathrm{ng} / \mathrm{ml}$ for SCC $(1.5 \mathrm{ng} / \mathrm{ml}$ in normal individuals), $447 \mathrm{U} / 1$ for TPA (110 U/1 in normal individuals). There were five tumor markers which had the same cut-off value in HD patients and in normal individuals: AFP, CA 125, PAP, PA and ST-439. The results couldn't establish a correlation between the tumor markers concentrations and BUN or serum creatinine. (11)

A study realized in 2007 by M. Polenakovic et al measured the pre-dialysis serum levels of several tumor markers in chronic HD patients. MCA, PSA, CA 125 and AFP had average concentrations within the normal range. The concentrations of CEA and CYFRA 21-1 were at the upper limit of the normal range. The mean concentrations of NSE and CA 19-9 were at least twice as high as normal levels. The authors concluded that the tumor markers' values in chronic HD patients should be interpreted with caution because of the impaired clearance by the hemodialysis membrane and that the imaging techniques are the best method for monitoring the acquired renal cystic disease that has a possible neplastic alteration. (12)

A study realized in 1988 by Walz G. et al included 93 patients on chronic dialysis. $92.5 \%$ of them had increased levels of TPA, $29.8 \%$ of them had increased levels of CEA and $6.5 \%$ of them had increased levels of AFP. There was found a significant correlation $(p<0.005)$ between the presence of increased TPA concentrations and of increased beta2-microglobulin concentrations. The results proves that some tumor markers can't be used for the management of malignant diseases in hemodialysis patients. (13)

A tumor marker that is frequently measured in dialysis patients is ferritin. It is useful as a reflection of the iron stores, an acute-phase reactant and an indicator of morbidity and mortality. A study realized by K. Kalantar-Zadeh et al, which included 101 adult hemodialysis patients, demonstrated that an increase in serum ferritin could represent a short-term indicator of death over a 12 -month period and that serum albumin could be a long-term indicator of mortality in hemodialysis patients. (14)

A study realized in Japan in 1992 included 31 hemodialysis patients without malignancies. The authors measured their concentrations of PAP (prostaticacidphosphatase), $\gamma$-Sm $(\gamma$-seminoprotein) 
and PSA (prostatic specific antigen). They were found to be similar to those of the control group and did not decrease after the dialysis treatment. The conclusion of the study was that they could be used for the diagnosis of prostatic cancer in HD patients. (15)

A study realized in 1991 by E. Odagiri et al measured the levels of 7 tumor markers: CEA, AFP, SCC, NSE, CA 125, CA 19-9 and CA 15-3 before and after the HD process in 144 uremic patients without malignant diseases. Before dialysis, SCC was the only tumor marker whose average concentration was increased. $95.1 \%$ of the patients had high levels of SCC, $25.7 \%$ of the patients had high levels of CEA and $10.6 \%$ of the patients had increased levels of NSE. All the patients had normal AFP concentrations. $7.6 \%$ of the patients had increased levels of $\mathrm{Ca} 125,6.3 \%$ of the patients had increased levels of CA $19-9$ and $3.5 \%$ of the patients had increased levels of CA 15-3. All the concentrations and also the total protein concentration increased after HD. The authors explained this fact by the incapacity of the membrane filter in the removal of tumor markers and concluded that their levels should be interpreted with caution. (16)

A study realized by Zeferos N. et al in 1991 included 50 healthy volunteers, 23 patients on chronic hemodialysis and 30 successfully transplanted individuals and measured the concentrations of AFP, CEA, CA 19-9, CA 125 and CA 15-3. The conclusion was that CA 125 and CA 19-9 could be successfully used in HD patients and patients after kidney transplantation. CEA and AFP had increased levels in chronic hemodialysis patients and normal concentrations in transplanted patients, thus the kidney tissue could have a role in their clearance. An unexplained fact was the elevation of CA 15-3 levels after the kidney transplantation (17).

A study realized in 1996 by N. Arik et al included 35 uraemic long-term haemodialysis patients, 35 pre-dialysis patients and 35 healthy controls. Neither of them had any sign of neoplasia. The authors measured the serum concentrations of AFP, CEA, CA 19-9, CA 125, PSA and SCC by means of the microparticle enzyme immunoassay. The results showed that the values of the tumor markers were similar in the first two groups of patients, the serum concentrations of SCC, CA 19-9 and CA 125 were significantly higher in the first two groups as compared to the control group and the serum concentrations of AFP, CEA and PSA were similar in the three groups. (7)

A study realized in 2005 included 232 non-dialysis patients diagnosed with chronic kidney disease and 37 uraemic patients on hemodialysis. The results showed a negative correlation between the creatinine clearance and the serum concentrations of CA 19-9, CA 125 (male), CYFRA 21-1, NSE and SCC-Ag. The levels of CA 125 (female), AFP, CA 15-3 and CA 72.4 were not influenced by the creatinine clearance. The patients treated by hemodialysis and the non-dialysis controls had similar values of CA 19-9, CA 125, AFP, CA 15.3, CA 72.4, CYFRA 21-1, NSE and SCC-Ag. The conclusion of the study was that the specificity of these tumor markers was influenced by the presence of chronic renal disease and was not affected by hemodialysis. (18)

Given the increased risk of patients with endstage renal failure to develop prostate cancer, several studies investigated the influence of the hemodialysis process on the determinations of tPSA, fPSA and the f/t PSA. The study included 149 men and the authors measured these parameters before and after dialysis (after the use of high-flux and low-flux membranes in the serum and in the dialysis ultrafiltrate). The results showed a significant decrease only in the concentrations of fPSA and f/ tPSA and only in high-flux membranes only. The variations in the concentrations of tPSA and IPSA were independent of age, serum creatinine, blood urea nitrogen and dialysis evaluation parameters. The conclusions of the study are that tPSA can be used in the screening of prostate cancer in HD patients independently from the type of membrane and that fPSA can be used reliably only in patients on HD with low-flux membranes. (19)

Another study realized by Tzanakis I. et al in 2002, which included 63 HD patients and 729 normal subjects founded that both groups had similar percentages of abnormal concentrations of tPSA and f/tPSA ratio. It concluded that a possible postdialysis raise in the total PSA level is probably due to hemoconcentration. (20)

\section{CONCLUSIONS}

Nowadays, the age of the population treated by dialysis is rising continuously and this leads to a higher frequency of malignancies in these patients (the most frequently encountered are renal cell, thyroid and endocrine organs, cervical, uterine, prostate, liver, tongue cancer and multiple myeloma). The screening process must take into account the variations of each tumor marker in HD patients and also the malignancy risk factors, the expected survival and the transplant status. (21) 


\section{REFERENCES}

1. Lye W.C., Tambyah P., Leong S.O. et al. Serum tumor markers in patients on dialysis and kidney transplantation. Adv Perit Dial. 1994; 10:109-111.

2. Pecchio F., Rapellino M., Ruffini E. et al. Half-life of carcinoembryonic antigen following curative resection of adenocarcinoma of the lung. Int J Biol Markers. 1992; 7:119-120.

3. Rapellino M., Piantino P., Pecchio F. et al. Disappearance curves of tumor markers after radical surgery. Int J Biol Markers. 1994; 9:33-37.

4. Tzitzikos G., Saridi M., Filippopoulou T. et al. Measurement of tumor markers in chronic hemodialysis patients. Saudi J Kidney Dis Transpl. 2010; 21:50-53.

5. Nomura F., Koyama A., Ishijima M. et al. Serum levels of five tumor markers for lung cancer in patients with chronic renal failure. Oncol Rep. 1998; 5:389-392.

6. Docci D., Pistocchi E., Turci F. et al. Serum CA 19-9 and CA 50 antigens in hemodialysis patients. Clin Nephrol. 1987; 27:179-181.

7. Port F.K., Ragheb N.E., Schwartz A.G. et al. Neoplasms in dialysis patients: a population-based study. Am J Kidney Dis. 1989; 14:119-123.

8. Cases A., Filella X., Molina R. et al. Tumor markers in chronic renal failure and hemodialysis patients. Nephron. 1991; 57:183-186.

9. Lye W.C., Tambyah P., Leong S.O. et al. Serum tumor markers in patients on dialysis and kidney transplantation. Adv Perit Dial. 1994; 10:109-111.

10. Sevinc A., Buyukberber S., Sari R. et al. Elevated serum CA-125 levels in hemodialysis patients with peritoneal, pleural, or pericardial fluids. Gynecol Oncol. 2000; 77:254-257.

11. Yiannakou L., Loucari-Yiannakou E., Souvatzoglou A. Digoxin-like immunoreactivity in cord blood plasma extracts is not only due to endogenous corticosteroids. Clin Biochem. 1991; 24:475-482.

12. Polenakovic M., Sikole A., Dzikova S. et al. Acquired renal cystic disease and tumor markers in chronic hemodialysis patients. Int $J$ Artif Organs. 1997; 20:96-100.
13. Walz G., Kunzendorf U., Keller F. et al. Elevated tumor markers in hemodialysis patients. Am J Nephrol. 1988; 8:187-189.

14. Kalantar-Zadeh K., Don B.R., Rodriguez R.A. et al. Serum ferritin is a marker of morbidity and mortality in hemodialysis patients. Am J Kidney Dis. 2001; 37:564-572.

15. Sasagawa I., Nakada T., Hashimoto T. et al. Serum prostatic acid phosphatase, gamma-seminoprotein and prostatic specific antigen in hemodialysis patients. Urol Int. 1992; 48:181-183.

16. Odagiri E., Jibiki K., Takeda M. et al. Effect of hemodialysis on the concentration of the seven tumor markers carcinoembryonic antigen, alpha-fetoprotein, squamous cell carcinoma-related antigen, neuron-specific enolase, CA 125, CA 19-9 and CA 15-3 in uremic patients. Am J Nephrol. 1991; 11:363-368.

17. Zeferos N., Digenis G.E., Christophoraki M. et al. Tumor markers in patients undergoing hemodialysis or kidney transplantation. Nephron. 1991; 59:618-620.

18. Xiaofang Y., Yue Z., Xialian X. et al. Serum tumour markers in patients with chronic kidney disease. Scand J Clin Lab Invest. 2007; 67:661-667.

19. Djavan B., Shariat S., Ghawidel K. et al. Impact of chronic dialysis on serum PSA, free PSA, and free/total PSA ratio: is prostate cancer detection compromised in patients receiving long-term dialysis? Urology. 1999; 53:1169-1174.

20. Tzanakis I., Kazoulis S., Girousis N. et al. Prostate-specific antigen in hemodialysis patients and the influence of dialysis in its levels. Nephron. 2002; 90:230-233.

21. Holley J.L. Screening, diagnosis, and treatment of cancer in long-term dialysis patients. Clin J Am Soc Nephrol. 2007; 2:604-610. 